\title{
INSTABILITY INTERVALS AND GROWTH RATES FOR HILL'S EQUATION
}

\author{
BY
}

\author{
JOSEPH B. KELLER
}

Departments of Mathematics and Mechanical Engineering, Stanford University, Stanford, California 94305-2125

Abstract. Hill's equation is a real linear second-order ordinary differential equation with a periodic coefficient $f(t)$ :

$$
y^{\prime \prime}(t)+[\lambda+\varepsilon f(t)] y(t)=0 .
$$

It has unbounded solutions for certain intervals of the real parameter $\lambda$, called instability intervals. Here these intervals, and the growth rate of the unbounded solutions, are determined for $\varepsilon$ small, and also for $\lambda$ large. This is done by constructing a fundamental pair of solutions which are power series in $\varepsilon / \lambda^{1 / 2}$, with coefficients that are bounded functions of $\lambda$.

1. Introduction. Hill's equation [1] is a real homogeneous linear second-order ordinary differential with a periodic coefficient. It can be written in the form (0.1) in which $\lambda$ and $\varepsilon$ are real parameters. The function $f(t)$ is a real periodic function of $t$ with minimal period $\pi$ and Fourier series

$$
f(t)=\sum_{n=-\infty}^{\infty} f_{n} e^{2 i n t}, \quad f_{-n}=\bar{f}_{n} .
$$

It is well known that there are intervals of $\lambda$, called intervals of instability, throughout which (0.1) has unbounded solutions. Our goal is to determine the locations and widths of these intervals, and the growth rates of the unbounded solutions, when $\varepsilon$ is small or when $\lambda$ is large. Then there is an instability interval of width $L_{m}$, near each value $\lambda=m^{2}$, where $m$ is a positive integer.

When $f(t)=\cos 2 t,(0.1)$ is the Mathieu equation. For it, Hale [2] showed that $L_{m}=O\left(\varepsilon^{m}\right)$, and Levy and Keller [3] found that

$$
L_{m}=\frac{8}{[(m-1) !]^{2}}\left(\frac{\varepsilon}{8}\right)^{m}[1+O(\varepsilon)], \quad f(t)=\cos 2 t
$$

Received June 28, 2007.

2000 Mathematics Subject Classification. Primary 34B30.

E-mail address: keller@math.stanford.edu 
Later Harrell [4] showed that for $m$ large, $L_{m}$ is given by (1.2) up to a constant factor with $O(\varepsilon)$ replaced by $O\left(\mathrm{~m}^{-2}\right)$. Avron and Simon [5] showed that the factor is one.

For Hill's equation in general, we shall show that

$$
L_{m}=2\left|\varepsilon f_{m}\right|+O\left(\varepsilon^{2} / m^{2}\right) .
$$

Thus $L_{m}$ does not decrease like $\varepsilon^{m}$, as it does for the Mathieu equation. Previously Erdelyi [6] obtained (1.3) for $m$ large, with $O\left(\varepsilon^{2} / m^{2}\right)$ replaced by $O\left(1 / m^{2}\right)$. We shall also show that within and near the $m$-th instability interval, the Floquet exponent $\alpha$ is given by

$$
\alpha=m-\frac{i}{2 m}\left[\left|\varepsilon f_{m}\right|^{2}-\left(\lambda-m^{2}+\varepsilon f_{0}\right)^{2}+O\left(\varepsilon^{3} / m\right)\right]^{1 / 2} .
$$

The growth rate of the solution is $|\operatorname{Im} \alpha|$, so when the square root in (1.4) is real, the solution grows exponentially with $t$. The endpoints of the $m$-th instability interval are the values of $\lambda$ at which the square root vanishes. Thus, if $f_{m} \neq 0$, the $m$-th instability interval is given by

$$
m^{2}-\varepsilon f_{0}-\left|\varepsilon f_{m}\right|+O\left(\frac{\varepsilon^{2}}{m}\right)<\lambda<m^{2}-\varepsilon f_{0}+\left|\varepsilon f_{m}\right|+O\left(\frac{\varepsilon^{2}}{m}\right) .
$$

In section 2 we construct a fundamental pair of solutions of $(0.1)$ in powers of $\varepsilon / \lambda^{1 / 2}$ and show that the coefficients are bounded functions of $\lambda$. Using these solutions, we define the discriminant $\Delta(\varepsilon, \lambda)$ and show that it is given by a power series in $\varepsilon / \lambda^{1 / 2}$. In section 3 we use the series for $\Delta$ to get the Floquet exponent $\alpha$ and to find the endpoints of the instability intervals.

2. Fundamental solutions and discriminant. Two linearly independent solutions of $(0.1), y_{1}(t)$ and $y_{2}(t)$, are determined by the initial conditions

$$
y_{1}(0)=1, \quad y_{1}^{\prime}(0)=0 ; \quad y_{2}(0)=0, \quad y_{2}^{\prime}(0)=1 .
$$

They satisfy the integral equations

$$
\begin{aligned}
& y_{1}(t)=\cos \lambda^{1 / 2} t-\frac{\varepsilon}{\lambda^{1 / 2}} \int_{0}^{t} y_{1}(s) f(s) \sin \left[\lambda^{1 / 2}(t-s)\right] d s, \\
& y_{2}(t)=\lambda^{-1 / 2} \sin \lambda^{1 / 2} t-\frac{\varepsilon}{\lambda^{1 / 2}} \int_{0}^{t} y_{2}(s) f(s) \sin \left[\lambda^{1 / 2}(t-s)\right] d s .
\end{aligned}
$$

When $f(s)$ is bounded and piecewise continuous, both of these equations can be solved by Picard iteration. This yields the following convergent series in powers of $\varepsilon / \lambda^{1 / 2}$ for $y_{1}$ and $y_{2}$ :

$$
y_{k}(t)=\sum_{j=0}^{\infty}\left(\frac{\varepsilon}{\lambda^{1 / 2}}\right)^{j} y_{k j}(t, \lambda), \quad k=1,2 .
$$


The coefficients $y_{k j}(t, \lambda)$ in $(2.4)$ are defined recursively:

$$
\begin{aligned}
y_{10}(t, \lambda) & =\cos \lambda^{1 / 2} t, \quad y_{20}(t, \lambda)=\lambda^{-1 / 2} \sin \lambda^{1 / 2} t \\
y_{k, j+1}(t, \lambda) & =-\int_{0}^{t} y_{k j}(s, \lambda) f(s) \sin \left[\lambda^{1 / 2}(t-s)\right] d s, k=1,2 ; j=0,1, \ldots
\end{aligned}
$$

For $t$ real and $\lambda \geq 0$, so that $\lambda^{1 / 2}$ is real, both $y_{10}$ and $y_{20}$ are bounded functions of $\lambda$. It follows from $(2.6)$ that if $y_{k j}(t, \lambda)$ is a bounded function of $\lambda$, then so is $y_{k, j+1}$. Therefore all the $y_{k j}$ are bounded functions of $\lambda$. Thus we have

TheOrem 2.1. Hill's equation (1.1) with $f(t)$ bounded and piecewise continuous has two solutions $y_{1}(t, \varepsilon, \lambda)$ and $y_{2}(t, \varepsilon, \lambda)$ satisfying the initial conditions (2.1). They are given by the convergent series (2.4) in powers of $\varepsilon / \lambda^{1 / 2}$. For $\lambda \geq 0$ the coefficients in (2.4) are bounded functions of $\lambda$.

The discriminant of Hill's equation, $\Delta(\varepsilon, \lambda)$, is defined by

$$
\Delta(\varepsilon, \lambda)=y_{1}(\pi, \varepsilon, \lambda)+y_{2}^{\prime}(\pi, \varepsilon, \lambda) .
$$

By using (2.4) for $y_{1}$ and $y_{2}$ in (2.7), we obtain for $\Delta(\varepsilon, \lambda)$ the following convergent series in powers of $\varepsilon / \lambda^{1 / 2}$ :

$$
\begin{aligned}
\Delta(\varepsilon, \lambda) & =\sum_{j=0}^{\infty}\left(\frac{\varepsilon}{\lambda^{1 / 2}}\right)^{j}\left[y_{1 j}(\pi, \lambda)+y_{2 j}^{\prime}(\pi, \lambda)\right] \\
& =2 \cos \lambda^{1 / 2} \pi+\sum_{j=1}^{\infty}\left(\frac{\varepsilon}{\lambda^{1 / 2}}\right)^{j}\left[y_{1 j}(\pi, \lambda)+y_{2 j}^{\prime}(\pi, \lambda)\right] .
\end{aligned}
$$

To show that $y_{2 j}^{\prime}(\pi, \lambda)$ is a bounded function of $\lambda$ for $\lambda \geq 0$, we observe from (2.5) that $y_{20}^{\prime}(t, \lambda)=\cos \lambda^{1 / 2} t$, which is bounded for $\lambda \geq 0$. We differentiate (2.6) to get

$$
y_{2, j+1}^{\prime}(t, \lambda)=\int_{0}^{t} y_{2 j}(s, \lambda) f(s) \frac{d}{d s} \sin \left[\lambda^{1 / 2}(t-s)\right] d s .
$$

Next we integrate by parts, assuming that $f(t)$ has a piecewise continuous derivative. Then (2.9) becomes

$$
y_{2, j+1}^{\prime}(t, \lambda)=\left.y_{2 j}(s, \lambda) f(s) \sin \left[\lambda^{1 / 2}(t-s)\right]\right|_{0} ^{t}-\int_{0}^{t}\left[y_{2 j}(s, \lambda) f(s)\right]^{\prime} \sin \left[\lambda^{1 / 2}(t-s)\right] d s .
$$

The first term on the right vanishes at $s=0$ because $y_{2 j}(0, \lambda)=0$, and it vanishes at $s=t$ because the sin vanishes. Thus $y_{2, j+1}^{\prime}(t, \lambda)$ is given by the integral in $(2.10)$. We have shown that $y_{2 j}$ in the integrand is bounded for $\lambda \geq 0$, and so is $y_{20}^{\prime}$. If $y_{2 j}^{\prime}(t, \lambda)$ is also bounded, then it follows that $y_{2, j+1}^{\prime}(t, \lambda)$ is bounded for $\lambda \geq 0$, so all $y_{2 j}^{\prime}(t, \lambda)$ are bounded for $\lambda \geq 0$.

Upon using this result and the boundedness of $y_{1 j}(t, \lambda)$, we have the following theorem: 
Theorem 2.2. Let $f(t)$ in (1.1) be bounded with a piecewise continuous derivative. Then the convergent series $(2.8)$ for $\Delta(\varepsilon, \lambda)$ in powers of $\varepsilon / \lambda^{1 / 2}$ has coefficients that are bounded functions of $\lambda$ for $\lambda \geq 0$.

It is often convenient to normalize $f(t)$ in $(0.1)$ by requiring that its integral over one period vanish. This can be accomplished by replacing $f$ and $\lambda$ by $\tilde{f}$ and $\tilde{\lambda}$ defined by

$$
\tilde{f}(t)=f(t)-f_{0} \quad, \quad \tilde{\lambda}=\lambda+\varepsilon f_{0} .
$$

The preceding analysis applies with these replacements, so Theorems 1 and 2 hold with these changes. Therefore we can use the results of Magnus and Winkler [1, p. 27, Corollary 26] to write the first three terms in the expansion (2.8). Their $\lambda$ is our $\tilde{\lambda}$ and their $g_{n}=\varepsilon \tilde{f}_{n}=\varepsilon f_{n}$ for $n \geq 1$, so we obtain

$$
\Delta(\varepsilon, \lambda)=2 \cos \pi \tilde{\lambda}^{1 / 2}+\left(\frac{\varepsilon^{2}}{\tilde{\lambda}}\right) \frac{\pi}{2} \tilde{\lambda}^{1 / 2} \sin \pi \tilde{\lambda}^{1 / 2} \sum_{n=1}^{\infty} \frac{\left|f_{n}\right|^{2}}{\tilde{\lambda}-n^{2}}+O\left[\left(\frac{\varepsilon}{\tilde{\lambda}^{1 / 2}}\right)^{3}\right] .
$$

Their error term $O\left(\varepsilon^{3}\right)$, and Theorem 2 above, show that the error term in (2.12) is $O\left[\left(\varepsilon / \tilde{\lambda}^{1 / 2}\right)^{3}\right]$.

3. Floquet exponent. The Floquet exponent $\alpha(\varepsilon, \lambda)$ is a solution of the equation

$$
\cos \pi \alpha(\varepsilon, \lambda)=\Delta(\varepsilon, \lambda) / 2=\cos \pi \tilde{\lambda}^{1 / 2}+\frac{\pi \varepsilon^{2}}{4 \tilde{\lambda}^{1 / 2}} \sin \pi \tilde{\lambda}^{1 / 2} \sum_{n=1}^{\infty} \frac{\left|f_{n}\right|^{2}}{\tilde{\lambda}-n^{2}}+O\left[\left(\varepsilon / \tilde{\lambda}^{1 / 2}\right)^{3}\right] .
$$

The second form of (3.1) follows from (2.12). When $\Delta \neq \pm 2, \alpha$ is not an integer. Then (0.1) has two linearly independent solutions of the forms

$$
y^{ \pm}(t)=e^{ \pm i \alpha t} p^{ \pm}(t), \quad \Delta \neq \pm 2 .
$$

Both $p^{+}$and $p^{-}$are periodic with period $\pi$.

If $|\Delta|<2$, then (3.1) implies that $\alpha$ is real, so both $y^{+}$and $y^{-}$are bounded functions of $t$. However, if $|\Delta|>2$, then (3.1) shows that $\alpha$ is imaginary, so one of the two solutions in (3.2) grows exponentially in $t$ with growth rate $|\operatorname{Im} \alpha|$. From (3.1) we see that for $\varepsilon / \tilde{\lambda}^{1 / 2}$ small, $|\Delta|>2$ only if $\tilde{\lambda}^{1 / 2}$ is close to an integer $m$. Therefore to determine the growth rate we write $\tilde{\lambda}$ in the form

$$
\tilde{\lambda}=m^{2}+\varepsilon\left(\mu+f_{0}\right) .
$$

Here $\mu$ is a new parameter which shows how close $\lambda$ is to $m^{2}$, and therefore how close $\tilde{\lambda}$ is to $m^{2}+\varepsilon f_{0}$. Now from (3.3),

$$
\tilde{\lambda}^{1 / 2}=m+\varepsilon\left(\mu+f_{0}\right) /(2 m)+O\left(\varepsilon^{2} / m^{3}\right) .
$$

We use (3.4) in (2.12) to get

$$
\Delta(\varepsilon, \lambda)=2 \cos \pi m\left[1-\frac{1}{2}\left(\frac{\pi \varepsilon}{2 m}\right)^{2}\left\{\left(\mu+f_{0}\right)^{2}-\left|f_{m}\right|^{2}\right\}+O\left(\varepsilon^{3} / m^{3}\right)\right] .
$$


To solve (3.1) for $\alpha$, we set $\alpha=m+\alpha_{1}$. Then $\cos \pi \alpha=\cos \pi m \cos \pi \alpha_{1}$. By using this and (3.5) in (3.1), we get

$$
\cos \pi m \cos \pi \alpha_{1}=\cos \pi m\left[1-\frac{1}{2}\left(\frac{\pi \varepsilon}{2 m}\right)^{2}\left\{\left(\mu+f_{0}\right)^{2}-\left|f_{m}\right|^{2}\right\}+O\left(\varepsilon^{3} / m^{3}\right)\right] .
$$

Upon writing $\cos \pi \alpha_{1}=1-\left(\pi \alpha_{1}\right)^{2} / 2+O\left(\alpha_{1}^{4}\right)$ in (3.6), and solving for $\alpha_{1}$, we obtain

$$
\alpha_{1}=\frac{i}{2 m}\left[\left|\varepsilon f_{m}\right|^{2}-\left(\varepsilon \mu+\varepsilon f_{0}\right)^{2}+O\left(\varepsilon^{3} / m\right)\right]^{1 / 2} .
$$

Since $\alpha=m+\alpha_{1}$ and $\lambda=m^{2}+\varepsilon \mu$, we can rewrite (3.7) in the form (1.4).

The result (3.7) shows that for $\lambda$ near $m^{2}$, the growth rate $|\operatorname{Im} \alpha|=\left|\operatorname{Im} \alpha_{1}\right|$ is positive when $\lambda$ is in the interval given by (1.5), and it is zero outside that interval.

\section{REFERENCES}

[1] Magnus, W. and Winkler, S., Hill's Equation, Interscience Publishers, John Wiley, New York, 1966. MR0197830 (33:5991)

[2] Hale, J. K., On the behavior of solutions of linear periodic differential equations near resonance points, Contributions to the theory of Nonlinear Oscillations, Vol. 5, 1960, 55-89, Annals of Math. Studies, Princeton Univ. Press, Princeton. MR0141827 (25:5224)

[3] Levy, D. M. and Keller, J. B., Instability Intervals of Hill's Equation, Comm. Pure Appl. Math. 16 (1963), 469-476. MR0153914 (27:3875)

[4] Harrell, E. On the effect of the boundary conditions on the eigenvalues of ordinary differential equations, Amer. J. Math. supplement dedicated to P. Hartman, Johns Hopkins Univ. Press, Baltimore, 1981. MR648460 (83c:34031)

[5] Avron, J. and Simon, B., The asymptotics of the gap in the Mathieu equation, Ann. Physics 134 (1981), 76-84. MR626698 (82h:34030)

[6] Erdelyi, A., Ueber die freien Schwingungen in Kondensatorkreisen von veränderlichen Kapazitaet, Ann. Physik, Vol. 19, 1934, 585-622. 